\title{
An anteromedial approach to the temporal horn to avoid injury to the optic radiation fibers and uncinate fasciculus: anatomical and technical note
}

\author{
Jeroen R. Coppens, M.D., Kelly B. Mahaney, B.A., And Saleem I. Abdulrauf, M.D. \\ Division of Neurosurgery, Saint Louis University, St. Louis, Missouri
}

\begin{abstract}
Object. The aim of this study was to define an anteromedial approach to the temporal horn via a transsylvian approach to avoid injury to the optic radiation fibers as well as the uncinate fasciculus. This route was compared with standard surgical approaches to the temporal horn, and their relationship to the optic radiation and uncinate fasciculus was reviewed.

Methods. Three cadaveric brain specimens were prepared with freezing and thawing cycles according to the Klingler technique. Dissection was performed in a lateral-to-medial fashion with the help of wooden spatulas. Photographs were taken through the operating microscope at every level of the dissection. The dissection was continued until the optic radiation was encountered. Particular attention was paid to the relationship of the uncinate fasciculus with the optic radiation. An anteromedial transsylvian approach was defined to enter the temporal horn without injuring the optic radiation or the uncinate fasciculus.

Conclusions. A transsylvian anteromedial approach through the pyriform cortex at the level of the anterior and superior surface of the uncus enables a safe entry into the temporal horn without injury to the optic radiation fibers or the main part of the uncinate fasciculus.
\end{abstract}

\section{KEY WORDS - temporal lobe • optic radiation • uncinate fasciculus • fiber dissection}

Neurosurgeons commonly approach mesial temporal lobe structures for epilepsy surgery, tumor resection, or when dealing with vascular lesions. Morbidity associated with temporal lobe surgery consists mainly of visual field deficits ${ }^{3,4,7,11-13,18,21}$ and aphasias. ${ }^{5}$ Based on our recent publication, we concluded that the optic radiation fibers completely cover the superior and lateral walls of the temporal horn, and the lateral portion of the tip of the temporal horn. ${ }^{23}$ In the same study we found that the entire medial wall of the temporal horn is free of optic radiation fibers, except at the level at which these fibers arise from the lateral geniculate body. Our aim is to define a transsylvian approach to medial temporal lobe structures that limits cortical resection and spares the optic radiation and uncinate fasciculus to avoid the aforementioned morbidities.

Since the anatomical description by Meyer, ${ }^{14}$ optic radiation fibers have been known to be in close relationship with the temporal horn of the lateral ventricle. This has subsequently been confirmed by multiple authors. ${ }^{1,3,19,20,23,26,29}$ The exact anatomy of the optic radiation fibers, which extend from the lateral geniculate body to the calcarine sulcus, has been defined by the use of white matter tract dissection techniques such as the one described by Klingler. ${ }^{10}$ Other white matter tracts, such as the uncinate fasciculus ${ }^{2,9}$, 19,27 connecting the frontal and temporal lobes, have been described.

When performing a lateral-to-medial dissection of the temporal lobe by using the Klingler technique, the first layer to be removed is the superficial cortex. The next layer is formed by the $\mathrm{U}$ fibers, which are short association fibers interconnecting the different gyri and lying deep to the sulci. The most superficial large white matter tract then encountered is the superior longitudinal fasciculus. At that point, the cortex of the insula is still intact. The next step in the dissection consists of exposing uncinate fasciculus in the anterior part of the temporal lobe.

The optic radiation can be subdivided into three bundles (anterior, middle, and posterior). The anterior bundle, commonly referred to as the Meyer loop, has the greatest anterior curve, which extends toward the tip of the temporal horn after arising from the lateral geniculate body. The anterior bundle covers the roof and lateral wall of the anterior tip of the temporal horn of the lateral ventricle. It ends on the inferior bank of the calcarine fissure, and it contains the visual information from the superior visual fields. ${ }^{1}$

The central bundle makes a partial anterior curve toward the anterior temporal horn after originating from the lateral geniculate body. It then courses posteriorly within the sagittal stratum to the calcarine cortex and corresponds to the macular fibers. It is therefore found on the superior and lateral wall of the temporal horn.

The posterior bundle is composed of fibers that leave the lateral geniculate body to terminate in the upper bank of the calcarine fissure without making an anterior curve. These fibers contain the visual information from the inferior visual fields and are found on the lateral wall of the atrium of the lateral ventricle.

Optic radiation fibers have been noted to be present on 
the roof and on the posteromedial and lateral walls of the temporal horn of the lateral ventricle. ${ }^{1,23}$ The inferior wall of the temporal horn has consistently been described as being devoid of optic radiation fibers.

Visual field defects have been noted after surgery involving the temporal lobe for treatment of epilepsy, and after tumor or vascular surgery. The defects tend to be incongruous when the anatomical lesions are more anteriorly situated. ${ }^{7,13}$ The incongruity is explained by the ipsilateral eye usually being affected more than the contralateral eye because of the more anterior position of the ipsilateral eye fibers. ${ }^{7}$ In some studies congruous defects have been noted, but these are thought to affect geniculocalcarine fibers after they make their anterior loop on the roof of the temporal horn. ${ }^{3}$ Investigators have reported a mixture of congruous and incongruous lesions after temporal lobe resections. ${ }^{29}$

The visual field defects vary from a small homonymous defect to a full homonymous hemianopia, depending on the extent of the injury to the geniculocalcarine tract or whether a vascular injury to the anterior choroidal artery is associated with it. ${ }^{12}$ The most common visual field defect is a superior homonymous quadrantanopia with a sharp medial border along the superior vertical meridian and the sloping of its inferior border to varying degrees to the horizontal meridian. ${ }^{29}$ Most partial homonymous upper quadrantanopias are not perceived by patients. ${ }^{21}$ They are common when a lateral surgical approach is used through the superior or middle temporal gyrus and patients are later systematically tested with formal visual fields. Visual field loss can be severe enough for patients to be unable to qualify to drive in $50 \%$ of cases after an anterior temporal lobectomy. ${ }^{18}$ In some series, up to $83 \%$ of patients had a visual field defect after lateral approaches, and all were asymptomatic. ${ }^{12} \mathrm{~Pa}-$ tients are more commonly aware of their visual field defects when lesions are in the occipital compared with the temporal lobe. ${ }^{11}$

Based on the anatomy of the optic radiation, our aim was to define a surgical trajectory to the temporal horn that would stay anterior and medial to the optic radiation.

\section{MATERIALS AND METHODS}

We studied three formalin-fixed, frozen brains according to the method described by Klingler. ${ }^{10}$ The brains are first fixed in $4 \%$ formalin for 10 days, then frozen at $-10^{\circ} \mathrm{C}$ for 24 hours, and then thawed and refrozen for 24 hours. The sequence of freezing and thawing is repeated for three to five cycles. The brains are stored in a $4 \%$ formalin solution at room temperature between dissection sessions. Dissection is performed with the use of handmade spatulas fashioned from wooden tongue depressors. A lateral-to-medial dissection is performed as described by Türe, et al., ${ }^{26}$ until the sagittal stratum is encountered. Serial photographs are obtained through the operating microscope (NC4; Zeiss, Oberkochen, Germany). Special emphasis is placed on the study of the relationship of the uncinate fasciculus to the optic radiation.

In all three specimens, we found that using the transsylvian, pyriform transcortical, transuncal trajectory allows entry into the temporal horn while saving the optic radiation and the uncinate fasciculus (Figs. 1-7). In one specimen, the temporal horn was entered through an inferior 1-

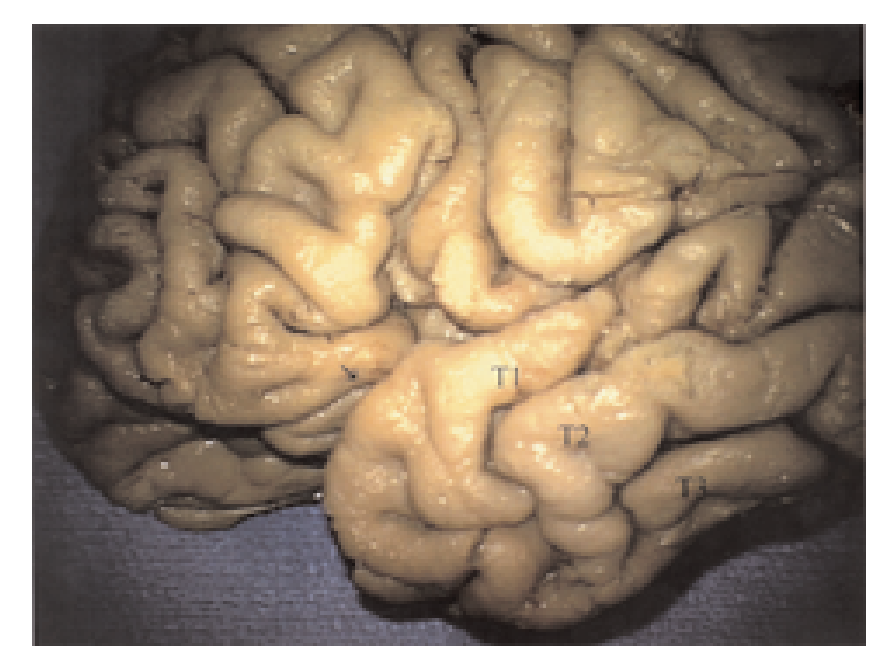

Fig. 1. Photograph showing a lateral view of a cadaveric brain prepared using the Klingler technique. $\mathrm{T} 1=$ superior temporal gyrus; T2 = middle temporal gyrus; T3 = inferior temporal gyrus; the arrow indicates the trajectory of a transsylvian approach.

$\mathrm{cm}$ incision made through the collateral sulcus (Fig. 3A). The lateral-to-medial dissection was performed until the sagittal stratum was encountered. A forceps was placed in the temporal horn with an anterior and medial trajectory confirming the location of our planned corticectomy in the pyriform cortex (Fig. 3B).

\section{ANATOMY AND APPROACH}

We used a Yaşargil pterional trjectory in these three specimens. The sylvian fissure was then dissected as described by Yaşargil and colleagues..$^{30,32}$ We recommend performing the dissection on the medial side of the veins in the sylvian fissure. The uncus and the anterior part of the parahip-

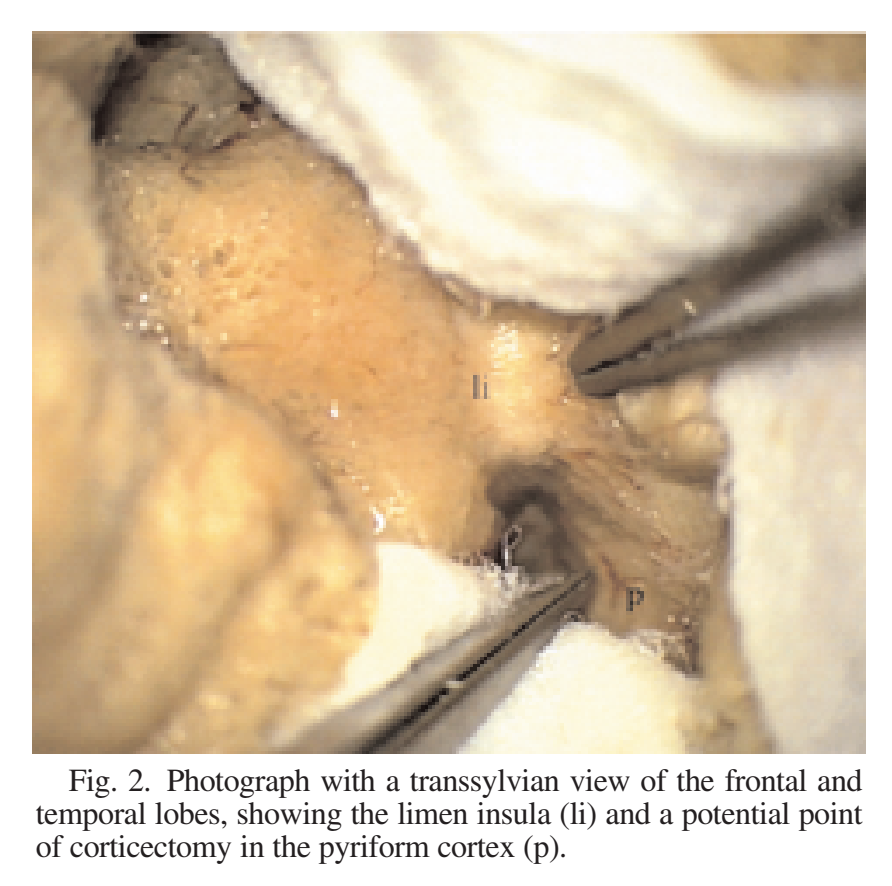



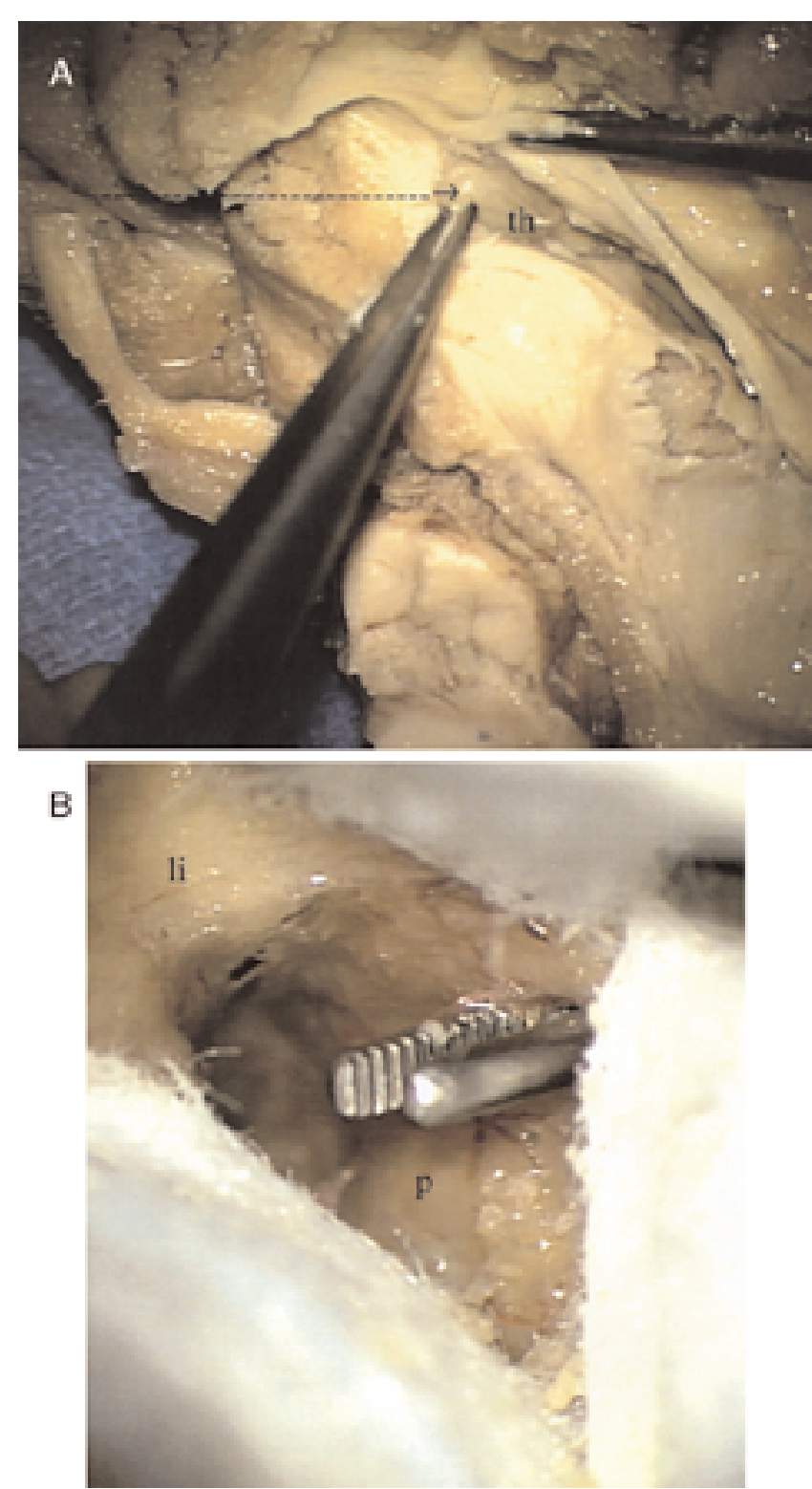

Fig. 3. A: Photograph showing an inferior view of a cadaveric brain after removal of cortex and exposure of the temporal horn (th) through an incision made through the collateral sulcus. A dotted line and arrow demonstrate the approach used for entry to the temporal horn as described in this paper. B: Photograph with a transsylvian view demonstrating the exit point on the parahippocampal gyrus ( $\mathrm{p}$ ) of a forceps inserted through the temporal horn inferiorly (as seen in panel A).

pocampal gyrus were identified. The medial surface of the uncus with its anterior and posterior part separated by their apex, as described by Rhoton, ${ }^{20}$ was identified. The pyriform cortex overlies the anterior pole of the parahippocampal gyrus as well as the uncus (Fig. 2). The amygdala lies just underneath the uncus (Fig. 5).

A corticectomy was performed in the sagittal plane in the pyriform cortex from its anterior pole to $3 \mathrm{~cm}$ posteriorly. The corticectomy was centered on the anterior-superior surface of the uncus (Fig. 3B). Just inferior to this, the rhinal sulcus was visualized, separating the uncus from the parahippocampal gyrus. The corticectomy was extended posteriorly to the level of the medial apex of the uncus. The corticectomy stayed anterior to the underlying uncinate fasciculus, which lies deep to the limen insula (Fig. 6). The amygdala was entered and partially removed in its medial and inferior components. The temporal horn of the lateral ventricle was subsequently entered by following a course just lateral and posterior to the amygdala (Fig. 7); the amygdala forms the anterior wall of the temporal horn. This trajectory was kept anterior and inferior to the uncinate fasciculus (Figs. 6 and 7). A small degree of injury to the uncinate fasciculus is possible when extending the resection laterally. The optic radiation fibers remained posterior to the uncinate fasciculus at all times and were therefore spared (Figs. 6 and 7).

\section{DISCUSSION}

Surgical approaches to the temporal horn of the lateral ventricle are commonly performed. Three surgical routes to the mesial temporal lobe structures and the temporal horn have commonly been described. They include a transsylvian approach, a lateral transsulcal or transcortical approach through the superior or inferior temporal sulcus and their adjacent gyri, and a subtemporal approach through the parahippocampal or fusiform gyri. The most frequently used route is the lateral transsulcal or transcortical approach.

The lateral approaches to the mesial temporal lobe involve varying degrees of resection through the superior, middle, or inferior temporal gyrus. ${ }^{25} \mathrm{~A}$ variant of these approaches consists of performing a corticectomy in the inferior temporal sulcus ${ }^{15,16}$ or the use of a transtemporal-transcisternal approach. ${ }^{8}$ The inferior temporal sulcus may be inconsistent and not identifiable in $28 \%$ of patients based on magnetic resonance imaging studies. ${ }^{16}$ When present, the majority of inferior temporal sulci have gyral bridges that make them noncontinuous, especially on the left side. ${ }^{16} \mathrm{~A}$

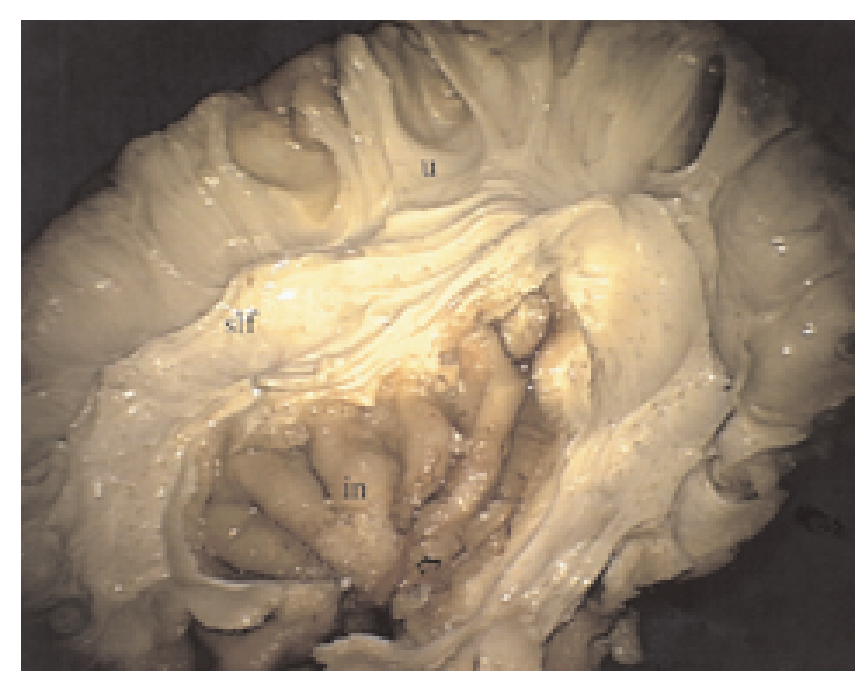

Fig. 4. Photograph showing a lateral view of a cadaveric brain after removal of cortex and superficial $U$ fibers $(\mathrm{u})$ by the Klingler technique. The arrow indicates the limen insula. in = insula; slf = superior longitudinal fasciculus. 


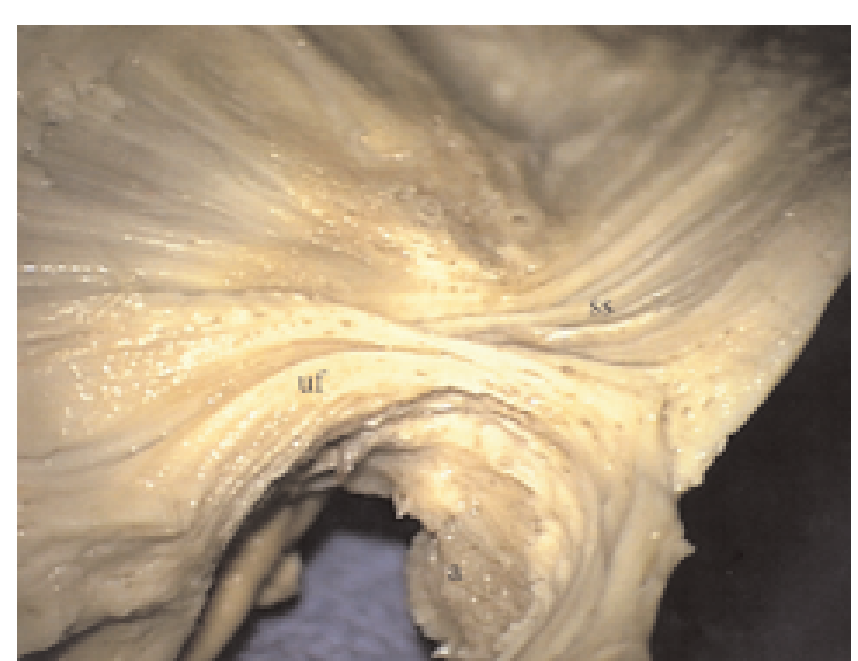

Fig. 5. Photograph with a transsylvian view of the specimen after removal of all layers until the uncinate fasciculus (uf) and sagittal stratum (ss) are exposed. $\mathrm{a}=$ amygdala.

wide degree of heterogeneity has been noted in regard to visual preservation with these approaches. Anatomical studies agree about the presence of optic radiation fibers along the lateral and superior wall of the temporal horn of the lateral ventricle but not the inferior wall. The medial wall contains some optic radiation fibers as they exit the lateral geniculate body. The inferior border of the optic radiation is topographically in accordance with the inferior temporal sulcus. In the group of lateral approaches, complete preservation of visual fields is best achieved by a corticectomy limited to stay inferior to the inferior temporal sulcus. ${ }^{15}$ The degree of visual field defects observed in these approaches is dependent on how superiorly the resection involves the lateral wall of the temporal horn. ${ }^{4}$

The second group of approaches is made via a subtemporal route. Corticectomies are performed through the parahippocampal ${ }^{17}$ or fusiform gyri. ${ }^{6}$ These approaches are very effective at visual field preservation because the entrance to the temporal horn is through its inferior wall, which is devoid of optic radiation fibers. Nevertheless, a significant degree of retraction has to be performed to give adequate access to the fusiform or parahippocampal gyrus, and this can lead to contusions or injury to the vein of Labbé. Performing a zygomatic osteotomy can limit the degree of retraction needed. ${ }^{22}$

A variation of an inferior approach to the temporal horn involves a supratentorial infraoccipital route when dealing with posteromedial lesions of the temporal horn. ${ }^{24}$ This route allows excellent results in terms of vision preservation but cannot address lesions that extend anteriorly. Retraction of the occipital lobe is required, which can cause transient visual defects.

The third group consists of transsylvian approaches. The sylvian fissure is dissected from the carotid bifurcation (medial to the sylvian veins) to the middle cerebral artery bifurcation and $2 \mathrm{~cm}$ beyond, exposing the anterior part of the insula and $\mathrm{M}_{2}$ segments. The $\mathrm{M}_{1}$ branches are then identified, with special attention paid to the anterior temporal and temporopolar arteries. A 2-cm corticectomy in the sup-

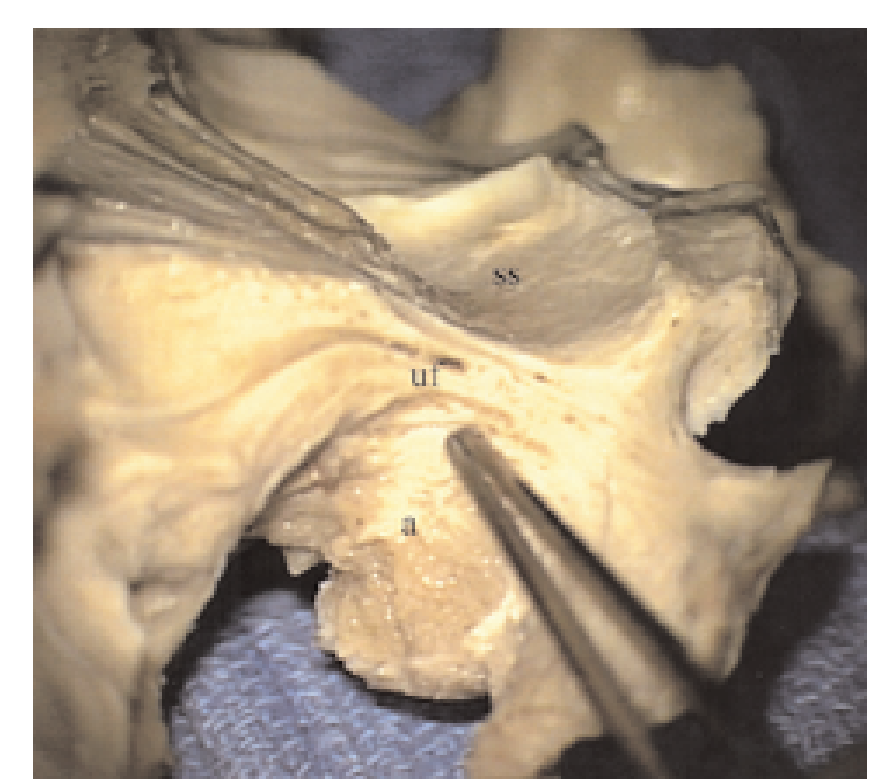

Fig. 6. Photograph with a transsylvian view showing the described approach used to enter the temporal horn while staying inferior to the uncinate fasciculus.

erior temporal gyrus is performed lateral to $\mathrm{M}_{1}$ and anteromedial to the $\mathrm{M}_{2}$ opening in the anterior portion of the uncinate fasciculus. The incision lies between the temporopolar and anterior temporal arteries.

These transsylvian approaches have been pioneered and described by Professor Yaşargil..$^{30-32}$ The amygdala is first entered superiorly after the corticectomy. The temporal horn can easily be entered in its tip by then taking an occipital trajectory. This procedure requires an expert knowledge of the anatomy of the region, and the whole resection is performed through a limited corticectomy. An amygdalohippocampectomy can be done from a lateral-to-medial

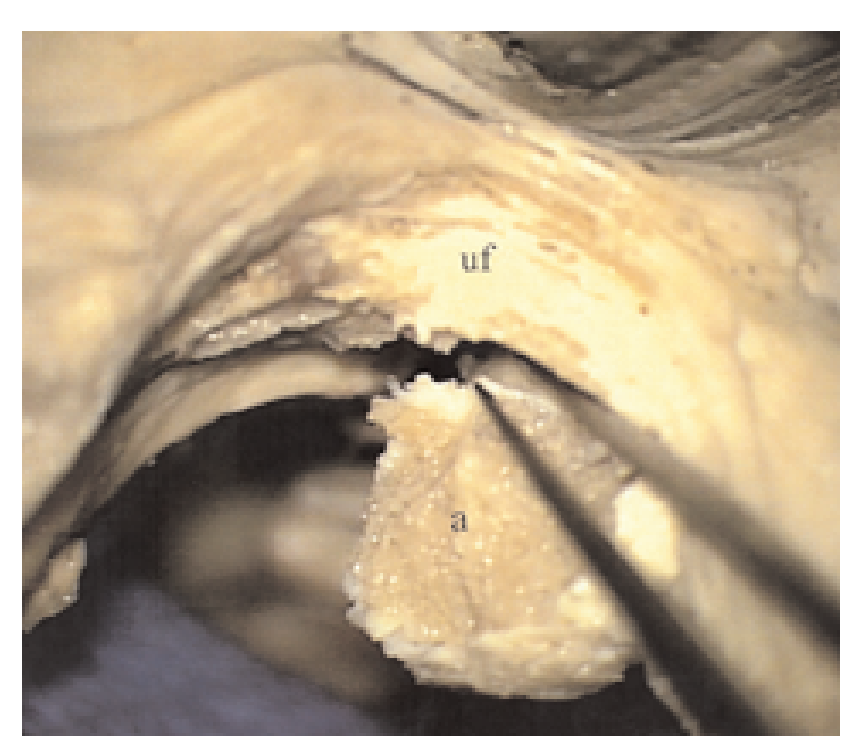

Fig. 7. Photograph showing a transsylvian view of the temporal horn from an approach that stays inferior to the uncinate fasciculus. 
direction (inside-out). A variant of the transsylvian approach is the transsylvian-transcisternal approach..$^{28}$ In this procedure, the ambient cistern is opened after performing the dissection of the sylvian fissure. An amygdalohippocampectomy is performed en bloc from a medial-to-lateral direction (outside-in). A temporary third nerve palsy was observed in $9 \%$ of cases, and 3\% of patients had a field defect postoperatively, as demonstrated by Goldmann perimetry. ${ }^{28}$

We believe a transsylvian approach to the mesial temporal lobe structures has the best chance of limiting visual field cuts and does not necessitate the use of retraction. All lateral approaches risk injuring the optic tract radiation fibers, even with tailored anterior lobectomies. The rostral extent of the Meyer loop varies considerably, which explains certain visual field defects that have been observed after resections limited to the anterior $3 \mathrm{~cm}$ from the temporal lobe tip..$^{12,13}$

Our proposed transsylvian, pyriform transcortical, transuncal trajectory is more medial and inferior than the corticectomy pioneered by Yaşargil in the transsylvian approach. Our approach uses more of a horizontal trajectory in the axial plane at the level of the temporal horn after starting the corticectomy at the pyriform cortex. The main trajectory of the subsequent dissection is posterior and lateral in that axial plane. The main trajectory described by Yaşargil, after the corticectomy through the superior temporal gyrus, is primarily inferior in the sagittal plane. The trajectory described in this paper allows us from an anatomical standpoint to avoid the main part of the uncinate fasciculus. The proposed approach in this paper is purely anatomical and has not been substantiated in the clinical setting. Yaşargil's approach has been validated in the clinical setting by his impressive series." ${ }^{31}$

\section{CONCLUSIONS}

We have defined a transsylvian trajectory that allows entry into the temporal horn while sparing the optic radiation and in all likelihood the main part of the uncinate fasciculus. This trajectory is accomplished through a corticectomy of the pyriform cortex at the level of the anterior part of the superior surface of the uncus. Further anatomical and clinical work needs to be conducted to substantiate this concept.

\section{References}

1. Ebeling U, Reulen HJ: Neurosurgical topography of the optic radiation in the temporal lobe. Acta Neurochir 92:29-36, 1988

2. Ebeling U, von Cramon D: Topography of the uncinate fascicle and adjacent temporal fiber tracts. Acta Neurochir 115:143-148, 1992

3. Falconer MA, Wilson JL: Visual field changes following anterior temporal lobectomy: their significance in relation to Meyer's loop of the optic radiation. Brain 81:1-14, 1958

4. Guenot M, Krolak-Salmon P, Mertens P, et al: MRI assessment of the anatomy of optic radiations after temporal lobe epilepsy surgery. Stereotact Funct Neurosurg 73:84-87, 1999

5. Henderson VW, Friedman RB, Teng EL, et al: Left hemisphere pathways in reading: inferences from pure alexia without hemianopia. Neurology 35:962-968, 1985

6. Hori T, Tabuchi S, Kurosaki M, et al: Subtemporal amygdalo- hippocampectomy for treating medically intractable temporal lobe epilepsy. Neurosurgery 33:50-57, 1993

7. Hughes TS, Abou-Khalil B, Lavin PJM, et al: Visual field defects after temporal lobe resection: a prospective quantitative analysis. Neurology 53:167-172, 1999

8. Ikeda K, Shoin K, Mohri M, et al: Surgical indications and microsurgical anatomy of the transchoroidal fissure approach for lesions in and around the ambient cistern. Neurosurgery 50: 1114-1120, 2002

9. Kier EL, Staib LH, Davis LM, et al: MR imaging of the temporal stem: anatomic dissection tractography of the uncinate fasciculus, inferior occipitofrontal fasciculus, and Meyer's loop of the optic radiation. AJNR 25:677-691, 2004

10. Klingler J: Erleichterung der makroskopischen Präparation des Gehirns durch den Gefrierprozess. Schweiz Arch Neurol Psychiatr 36:247-256, 1935

11. Koehler PJ, Endtz LJ, Te Velde J, et al: Aware or non-aware. On the significance of awareness for the localization of the lesion responsible for homonymous hemianopia. J Neurol Sci 75:255-262, 1986

12. Krolak-Salmon P, Guenot M, Tiliket C, et al: Anatomy of optic nerve radiations as assessed by static perimetry and MRI after tailored temporal lobectomy. Br J Ophthalmol 84:884-889, 2000

13. Marino R Jr, Rasmussen T: Visual field changes after temporal lobectomy in man. Neurology 18:825-835, 1968

14. Meyer A: The connections of the occipital lobes and the present status of the cerebral visual affections. Trans Assoc Am Physicians 22:7-16, 1907

15. Miyagi Y, Shima F, Ishido K, et al: Inferior temporal sulcus approach for amygdalohippocampectomy guided by a laser beam of stereotactic navigator. Neurosurgery 52:1117-1124, 2003

16. Miyagi Y, Shima F, Ishido K, et al: Inferior temporal sulcus as a site of corticotomy: magnetic resonance imaging analysis of individual sulcus patterns. Neurosurgery 49:1394-1398, 2001

17. Park TS, Bourgeois BF, Silbergeld DL, et al: Subtemporal transparahippocampal amygdalohippocampectomy for surgical treatment of mesial temporal lobe epilepsy. Technical note. J Neurosurg 85:1172-1176, 1996

18. Pathak-Ray V, Ray A, Walters R, et al: Detection of visual field defects in patients after anterior temporal lobectomy for mesial temporal sclerosis - establishing eligibility to drive. Eye 16: 744-748, 2002

19. Peuskens D, van Loon J, Van Calenbergh F, et al: Anatomy of the anterior temporal lobe and the frontotemporal region demonstrated by fiber dissection. Neurosurgery 55:1174-1184, 2004

20. Rhoton AL Jr: The cerebrum. Neurosurgery 51 (Suppl 4): S1-S51, 2002

21. Shahinfar S, Johnson LN, Madsen RW: Confrontation visual field loss as a function of decibel sensitivity loss on automated static perimetry. Ophthalmology 102:872-877, 1994

22. Shimizu H, Suzuki I, Ishijima B: Zygomatic approach for resection of mesial temporal epileptic focus. Neurosurgery 25: 798-801, 1989

23. Sincoff EH, Tan Y, Abdulrauf SI: White matter fiber dissection of the optic radiations of the temporal lobe and implications for surgical approaches to the temporal horn. J Neurosurg 101: 739-746, 2004

24. Smith KA, Spetzler RF: Supratentorial-infraoccipital approach for posteromedial temporal lobe lesions. J Neurosurg 82: 940-944, 1995

25. Spencer DD, Spencer SS, Mattson RH, et al: Access to the posterior medial temporal lobe structures in the surgical treatment of temporal lobe epilepsy. Neurosurgery 15:667-671, 1984

26. Türe U, Yaşargil MG, Friedman AH, et al: Fiber dissection technique: lateral aspect of the brain. Neurosurgery 47:417-426, 2000

27. Ungerleider LG, Gaffan D, Pelak VS: Projections from inferior 
temporal cortex to prefrontal cortex via the uncinate fascicle in rhesus monkeys. Exp Brain Res 76:473-484, 1989

28. Vajkoczy P, Krakow K, Stodieck S, et al: Modified approach for selective treatment of temporal lobe epilepsy: transsylvian-transcisternal mesial en bloc resection. J Neurosurg 88:855-862, 1998

29. Van Buren JM, Baldwin M: The architecture of the optic radiation in the temporal lobe of man. Brain 81:15-40, 1958

30. Wieser HG, Yaşargil MG: Selective amygdalohippocampectomy as a surgical treatment of mesiobasal limbic epilepsy. Surg Neurol 17:445-457, 1982

31. Yaşargil MG, Teddy PJ, Roth P: Selective amygdalo-hippocampectomy Operative anatomy and surgical technique. Adv Tech Stand Neurosurg 12:93-123, 1985
32. Yaşargil MG, Wieser HG, Valavanis A, et al: Surgery and results of selective amygdalo-hippocampectomy in one hundred patients with nonlesional limbic epilepsy. Neurosurg Clin $\mathbf{N}$ Am 4:243-261, 1993

Manuscript received April 22, 2005.

Accepted in final form May 25, 2005.

Address reprint requests to: Saleem I. Abdulrauf, M.D., Cerebrovascular and Skull Base Surgery Program, Division of Neurosurgery, Saint Louis University School of Medicine, 3635 Vista Avenue, St. Louis, Missouri 63110. 